$a=25 \cdot 12 \mathrm{kX} . ; b=14.65 \mathrm{kX}$.; (c) $26.79 \mathrm{kX}$.; $\beta=109 \cdot 7^{\circ} ; d(001)=25 \cdot 23 \mathrm{kX}$. Density (obs.) $=$ $1.44 \mathrm{gm} . / \mathrm{cm}^{3}$; density (ealc.) $=1.45 \mathrm{gm} . / \mathrm{cm}^{3}$ (assuming 32 molecules of $\left(\mathrm{CH}_{3} \cdot \mathrm{CH}_{2} \cdot \mathrm{CH}_{2} \cdot \mathrm{COO}-\right)_{2}$ Cu. $\mathrm{H}_{2} \mathrm{O}$ per unit cell).

The observed halvings were $\{h k l\}$ when $(k+l)$ is odd and $\{h 0 l\}$ all halved.

The space group is, therefore, $C_{8}^{4}-A a$ or $C_{2 h}{ }^{8}-$ $A 2 / a$. In the former space group, four asymmetric units are necessary, so that each unit would consist of eight molecules, whereas only four molecules per asymmetric unit are necessary in the latter space group. It is rather unusual for such a large number of molecules to be required.

I am indebted to Mr. Lang for preparing this compound, and to Dr. Vand and Mr. Lomer for carrying out the dehydration experiments. I wish to thank the director of the Chester Beatty Research Institute of the Royal Cancer Hospital (Free) for the loan of some apparatus, and the directors of Lever Brothers and Unilever, Ltd., for permission to publish these results.

Research Department,

John IBal.

Lever Brothers and Unilever, Ltd.,

Port Sunlight, Cheshire.

Nov. 25.

${ }^{1}$ Groth, P. H., "Chemische Krystallographie”, 3, 245.

\section{Response of Photoconductive Lead Sulphide Cells}

IN a recent communication, the possibility of using photoconductive lead sulphide cells for the measurement of rapidly varying surface temperatures has been emphasized ${ }^{1}$. For this purpose it is, of course, desirable that the response time of the measuring instrument be sufficiently small, and it is therefore of interest to see whether on theoretical grounds it is possible to decrease the response time of the cells. In this connexion it is important to remember that the conduction electrons can be captured both by the ionized impurities ( $n$ per c.c.), the number of which increases with increasing temperature, and by interstitial ions. At ordinary temperatures the equilibrium number of the latter (Frenkel defects) is very small ; however, under suitable conditions of manufacture, a large number ( $M$ per c.c.) corresponding to a high-temperature equilibrium can be 'frozen-in'. The number of conduction electrons released by radiation ( $I$ per c,c. per sec.) and heat agitation ( $A$ per c.c. per sec.) is then determined by the equation

$$
\frac{d n}{d t}=I+A-B n(n+M),
$$

the capture coefficients of the ionized impurities and interstitial ions being assumed equal for the sake of simplicity. The time $t_{\alpha}$ taken to obtain the fraction $\alpha$ of the full asymptotic response is, therefore, given by

$$
2 B\left(n_{0}+\frac{1}{2} M\right) t_{\alpha}=\frac{1}{\delta} \log \frac{\delta+1+(\delta-1) \alpha}{(\delta+1)(1-\alpha)},
$$

where

$$
\begin{gathered}
\delta=\sqrt{1+\frac{I}{B\left(n_{0}+\frac{1}{2} M\right)^{2}}}= \\
\sqrt{1+\frac{\left(n_{\infty}-n_{0}\right)\left(n_{\infty}+n_{0}+M\right)}{\left(n_{0}+\frac{1}{2} M\right)^{2}}} ; \\
n_{0}=n(t=0), \quad n_{\infty}=n(t=\infty) .
\end{gathered}
$$

Hence, if $M \gg n$,

$$
t_{\alpha}=\frac{1}{B M} \log \frac{1}{1-\alpha},
$$

whatever $I$ may be.

On the other hand, if $M \ll n$,

$$
\begin{gathered}
t_{\alpha}=\frac{1}{2 B n_{0}} \log \frac{1}{1-\alpha} \text { for small } \delta-1, \\
t_{\alpha}=\frac{1}{2 B n_{0} \delta} \log \frac{1}{1-\alpha}=\frac{1}{2 \sqrt{I B}} \log \frac{1}{1-\alpha} \text { for }
\end{gathered}
$$

large $\delta-1$.

As $B$ varies probably only slowly with temperature, the first formula shows that in the case $M \gg n$ the response time is almost independent of temperature but varies inversely as $M$. As distinct from this case, the response time decreases exponentially with increasing temperature of the cell if $M \ll n$, owing to the exponential increase of $n_{0}$ for semi-conductors. The case of large $\delta-1$ requires too high intensities of radiation to be important in practice.

It is to be expected that the case of a large number of 'frozen-in' capturing centres will be realized in practice if there is a large and rapid drop in the diffusion coefficient of the Frenkel defects during the manufacture of the cell ${ }^{2}$; for example, if the lead sulphide film is obtained by evaporation at a high temperature and sublimation on a cool support. The higher the temperature at which the film is formed, and the cooler the support, the larger $M$ will be, that is, the smaller the response time $t_{\alpha}$. The other case, $M \ll n$, can be expected to apply to cells made at ordinary temperatures, for example, by chemical precipitation.

It must be pointed out that the decrease in response time is accompanied by a corresponding decrease in response (signal), the asymptotic number of photoelectrons being given by

$$
n_{\infty}-n_{0}=\frac{I}{2 B\left(n_{0}+\frac{1}{2} M\right)}=\frac{I t_{\alpha}}{-\log (1-\alpha)} .
$$

Conversely, the formula shows that, at a loss of speed of response, the sensitivity of the cells can be increased by the methods indicated.

Details and comparison with experiments will be given elsewhere. I have to thank the Director-General of Scientific Research (Defence), Ministry of Supply, for permission to publish the present communication. W. A. Hepner

Department of Mathematics,

Imperial College of Science and Technology, London, S.W.7. Oct. 17.

${ }^{1}$ Lee, E., and Parker, R. C., Nature, 158, 518 (1946).

2 Mott and Gurney, "Electronic Processes in Ionic Crystals" (Oxford, 1940 ).

\section{Temperature Dependence of the Intensities of Raman Lines}

I HAVE undertaken the critical and exhaustive study of Placzek's polarizability theory of Raman scattering. My investigations have been carried out both with solids and liquids, and the following observations made: (1) The intensity of the Stokes lines decreases with the rise of temperature, while according to the theory there should be an increase. (2) The intensity of the anti-Stokes lines, in general, increases with the increase of temperature, but not to the extent that would be expected from Placzek's theory. (3) The intensities of the Stokes and the 